\title{
Flächenbewegliche Fahrantriebe für moderne Materialfluss- elemente am Beispiel von KARIS
}

\author{
Dipl.-Ing. Christoph Nobbe, Dipl.-Ing. Tobias Baur, Dr.-Ing. Frank Schönung, \\ Prof. Dr.-Ing. Kai Furmans \\ KIT - Karlsruher Institut für Technologie \\ Institut für Fördertechnik und Logistiksysteme \\ Fachbereich Fördertechnik
}

Abstract: Die Anforderungen an Materialflusselemente befinden sich im Wandel. Zum einen werden hohe Durchsätze und hohe Einzelverfügbarkeiten gefordert und zum anderen eine hohe Flexibilität sich auf schnell wechselnde Anforderungen einstellen zu können.

Am KIT ist, in enger Zusammenarbeit mit der Industrie, das Projekt KARIS entstanden. Dabei handelt es sich um dezentral gesteuerte Einzelelemente welche intralogistische Aufgaben ausführen können. Die Elemente agieren entweder als Einzelmodul, oder bilden Funktionscluster um größere Aufgaben übernehmen zu können. Eine typische Aufgabe für ein Einzelmodul ist der Transport eines Kleinladungsträgers $(K L T)$ über eine bestimmte Strecke. Die Funktionscluster unterteilt man in Unstetig- und Stetigförderer. Schließen sich z.B. vier Einzelelemente zusammen um gemeinsam als größeres FTS eine Palette zu befördern ist dies ein Unstetigcluster. Ein typisches Szenario für einen Stetigförderer ist die temporäre Bildung eines Förderbandes aus mehreren Elementen um einen LKW mit Paketen zu be- oder entladen.

Dieses Einsatzspektrum stellt besondere Anforderungen an die Fahrwerke der Einzelelemente. Kann ein Element alleine noch Aufgaben mit einem standardisierten Fahrantrieb bewältigen, ist es im Verbund schon deutlich schwieriger. So ist es für eine starr gekoppelte Formationsfahrt, z.B. vier Elemente transportieren gemeinsam eine Palette, notwendig ein Fahrwerk mit mehr Freiheitsgraden zu verwenden. Ebenso profitiert auch das Einzelmodul von einer höheren Beweglichkeit. So fällt das Rangieren in engen Bereichen eines Lagers deutlich leichter, wenn auch eine Querfahrt oder das Wenden auf der Stelle möglich sind.

Die Gewährleistung dieser Funktionalität der Einzelmodule setzt einen flächenbeweglichen Antrieb, idealerweise einen holonomen Antrieb, voraus.

\section{$1 \quad$ Einleitung}

Immer noch gilt die Faustformel, dass etwa die Hälfte der Gesamtkosten einer fördertechnischen Anlage durch die Planung, Installation und die Inbetriebnahme verursacht werden (vgl. Abb. 1). Diese Kosten, plus die Kosten für die Ausfallzeiten der Anlage, stehen bei einer Umkonfiguration und Wiederinbetriebnahme erneut an. Daher wird ein Umbau oftmals gescheut. 


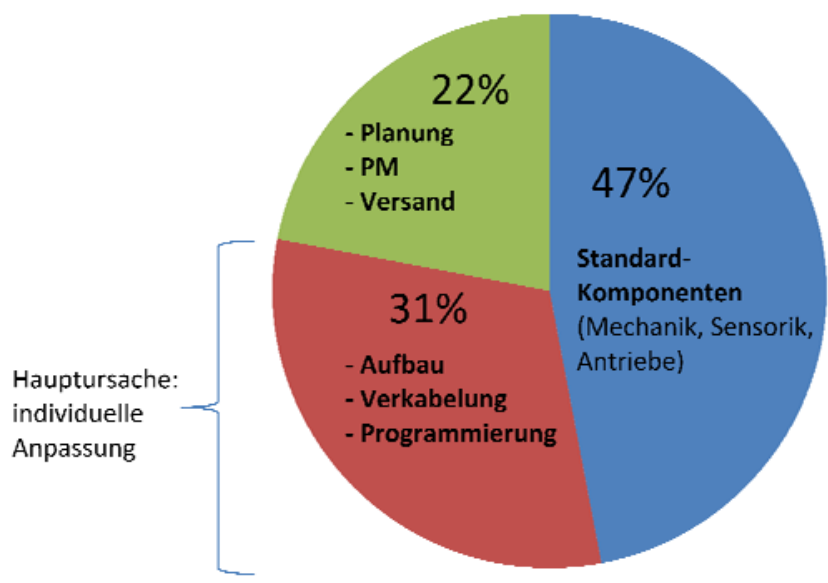

Abbildung 1: Kostenstruktur heutiger Stetigfördertechnik (nach psb Intralogistik GmbH, Typische Kostenstruktur für Stetigfördersysteme)

Dank virtueller Entwicklung wird die benötigte Zeit, um ein neues Modell auf den Markt zu bringen, immer kürzer, was gleichzeitig die Produktlebenszyklen verkürzt. Die dadurch kleiner werdenden Planungshorizonte und kürzer werdenden Amortisationszeiten lassen nur noch kleinere Investitionsvolumen zu. Dagegen steht der Wunsch der Kunden immer individuellere Produkte zu erwerben, was zu kleineren Losgrößen führt. Nicht auch zuletzt vor dem Hintergrund der Ressourcenschonung wird daher die Wandlungsfähigkeit von Produktionssystemen und Produktionsversorgungen immer wichtiger.

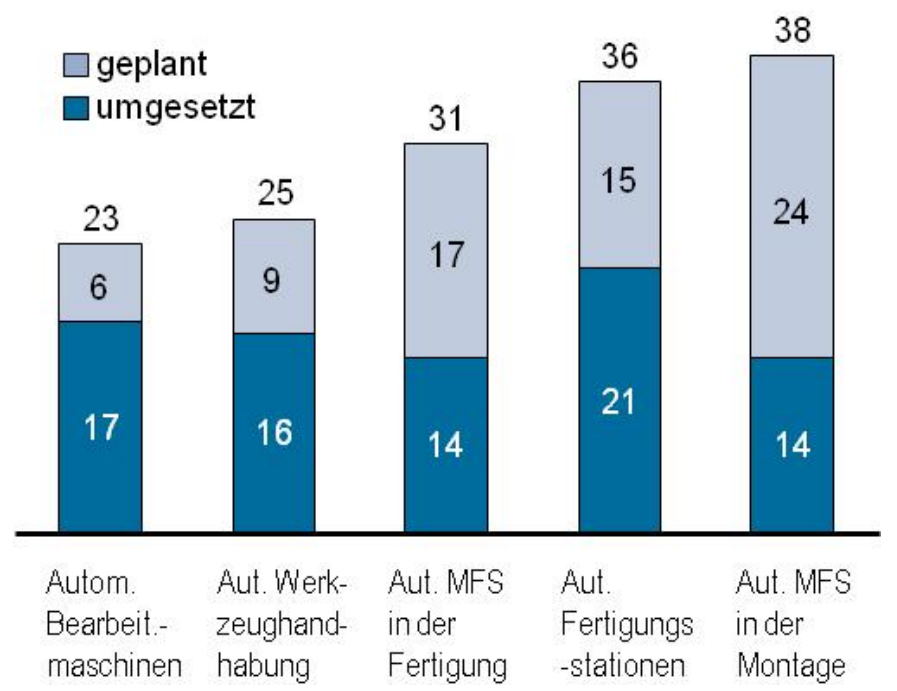

Abbildung 2: Reduktion des Automatisierungsgrades (laut Umfrage bei Nutzern hoch automatisierter Anlagen, VDI Nr. 35, 2001)

Während Anlagen bisher viele Jahre mit nahezu unverändertem Layout im Einsatz waren, ist es heute oftmals bereits nach wenigen Jahren - bei bestimmten Produkten sogar in noch kürzeren Zyklen - erforderlich, den Materialfluss neuen (c) 2010 Logistics Journal : Proceedings - ISSN 2192-9084 
Randbedingungen anzupassen. Im Bereich der Logistikdienstleister und produzierenden Unternehmen wird deshalb auf eine Automatisierung häufig teilweise oder gar vollständig verzichtet beziehungsweise der bereits vorhandene Automatisierungsgrad reduziert (siehe Abb. 2). Um auf die sich schnell ändernden Anforderungen der Märkte reagieren zu können, besteht die Notwendigkeit immer flexiblerer und wandelbarer Anlagen.

\section{KARIS}

KARIS (Abb. 3). Es wurde als skalierbares, rekonfigurierbares Materialflusssystem konzipiert und lässt sich flexibel ohne Einbußen der Betriebsbereitschaft auf alle Anforderungen anpassen. Grundlage dafür ist der modulare Aufbau. Das System besteht aus wenigen Grundelementen, welche in Kombination ein flexibles Gesamtsystem bilden. Eine Besonderheit von KARIS ist die völlig freie Navigation, wodurch dieses neue Verfahren ohne Eingriffe in die Umwelt auskommt. Ein Verteilen von Landmarken in Form von Funksendern, Reflektoren, Magneten, Bodenmarkierungen oder RFID-Tags, ist nicht notwendig. Vielmehr werden die natürlich vorhandenen Kanten und Flächen des Gebäudes für eine Navigation genutzt. Dies können Wände, Säulen oder auch die vorhandenen Maschinen sein. Anbindungen an bestehende Peripherie wie ein automatisches Kleinteilelager (AKL) oder andere Bereiche mit bereits installierter Fördertechnik werden als Übergabestelle definiert und können als Schnittstelle für den Materialfluss dienen. Ändert sich der Materialfluss aufgrund neuer Varianten oder Modelle, lassen sich die Zielstellen flexibel anpassen. Dazu ist es notwendig die neuen Koordinaten der Übergabestellen an die Einzelelemente zu übermitteln, was auch im laufenden Betrieb erfolgen kann.

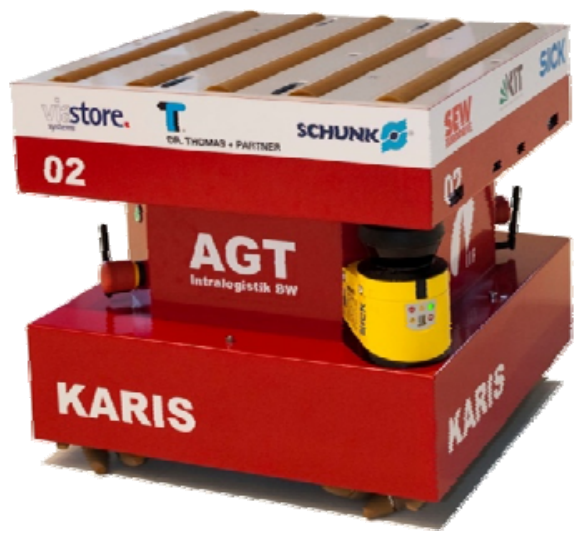

Abbildung 3: KARIS-Einzelelement

Der dezentrale Ansatz erlaubt es eine Aufstockung oder Reduzierung des Durchsatzes leicht vorzunehmen. Je nach Bedarf können mehr oder weniger Einzelelemente dem Gesamtsystem zur Verfügung gestellt werden. Da die Wegfindung, Auftragsvergabe und Koordination untereinander von den Elementen selbst erfolgt, ist kein aufwändiges umprogrammieren des Systems notwendig. Das System setzt sich aus der Anbindung an eine übergeordnete Peripherie, (c) 2010 Logistics Journal : Proceedings - ISSN 2192-9084 
verschiedene Übergabestellen (min. 2) und einer fast beliebigen Anzahl von Einzelelementen zusammen. Je nach Auftrag agiert KARIS dabei als Einzeltransporteur oder schließt sich zu einem Verbundsystem funktionaler Clustern zusammen. Der einfachste Fall ist der Transport eines Kleinladungsträgers von einem Punkt zum nächsten. Dies kann einfach und effizient als Einzeltransport durchgeführt werden. Je nach Auftragsaufkommen entscheidet das Gesamtsystem ob Einzeltransporte durchgeführt werden oder ob es notwendig wird ein funktionales Cluster zu bilden. In so einem Fall schließen sich mehrere Elemente zu einem Unstetigcluster zusammen um einen Großladungsträger zu transportieren, oder bilden selbständig eine temporäre Stetigförderstrecke um gezielt einen höheren Durchsatz zu erreichen (siehe Abb. 4). Diesen Zusammenschluss und die Entscheidung hierzu übernehmen die Elemente selbständig und entscheiden dezentral, welche Elemente für den jeweiligen Cluster benötigt werden.

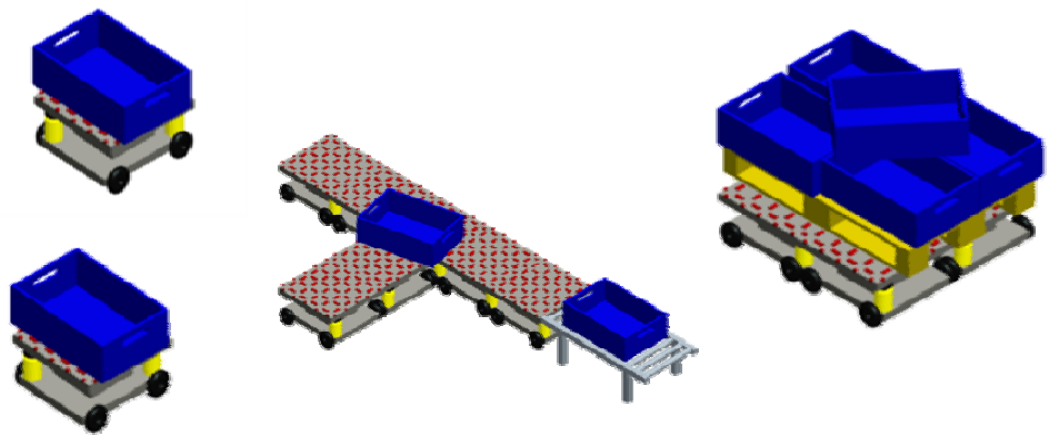

Abbildung 4: Einzelelemente und funktionale Cluster

Der standardisierte, modulare Aufbau der Einzelelemente ermöglicht es Ausfälle einzelner Module zu kompensieren, indem ein anderes, funktionsfähiges Modul die Aufgaben des ausgefallenen übernimmt. Dies stellt eine Redundanz sicher und die Herstellungskosten können durch höhere Stückzahlen gesenkt werden. Auch die Tatsache, dass die einzelnen Module keine äußerst hohe (99,5\% oder höhere) und damit sehr teure Verfügbarkeit mehr aufweisen müssen trägt zur Kostenreduktion bei.

\section{Anforderungen an die Fahrantriebe moderner Materialflusssysteme}

\subsection{Allgemein}

Betrachtet man die Flächenkosten verschiedener Distributionszentren mit Hilfe des DCRM (Distribution Center Reference Model) [1] werden Einsparpotenziale deutlich. Mit flächenbeweglichen Antrieben lassen sich Flächen, welche zum Rangieren genutzt werden und breite Kurvenpassagen einsparen, da aus dem Stand heraus alle Bewegungen möglich sind [9]. Da die Kosten pro Quadratmeter bis $140 €$ pro Jahr betragen, rechnet sich jede Einsparung in der Fläche. Desweiteren lassen sich Wegstrecken aufgrund besserer Fahrwege verkürzen. Ebenso wichtig sind hohe Dynamik und Geschwindigkeit. Neue Materialflusssysteme müssen sich in puncto (c) 2010 Logistics Journal : Proceedings - ISSN 2192-9084 
Leistung und Durchsatz mit herkömmlichen Anlagen messen lassen. Wichtig ist eine hohe Fahrstabilität, um bei unterschiedlichen Beladungen ein Aufschaukeln zu vermeiden.

\subsection{Anforderungen an die Fahrantriebe im Hinblick auf die Verbundfahrt}

Für Verbundfahrten wird es zusätzlich wichtig sehr schnell Richtungsänderungen durchführen zu können. Ein Warten auf Lenkbewegungen sorgt für Zwangspausen des Verbunds und reduziert damit die Leistungsfähigkeit. Genauso muss eine hohe Spurtreue gewährleistet sein, da es sonst zu erhöhtem Verschleiß kommt.

\subsection{Herausforderungen bei Verbundfahrten}

Um die notwendigen Bahnkurven der Fahrantriebe im Verbund zu verdeutlichen werden verschiedene Situationen einer solchen Fahrt simuliert. Die Ausgangssituation ist in allen Fällen die gleiche, nämlich der Transport einer Palette mit Hilfe von vier unabhängigen Fahrzeugen. Die Problemstellung lässt sich auch auf andere Fälle übertragen und steht stellvertretend für beliebige andere Fahrten im Transportverbund.

In der Ausgangssituation sind die Transportfahrzeuge gleichmäßig unter der Palette angeordnet (Abb. 5).

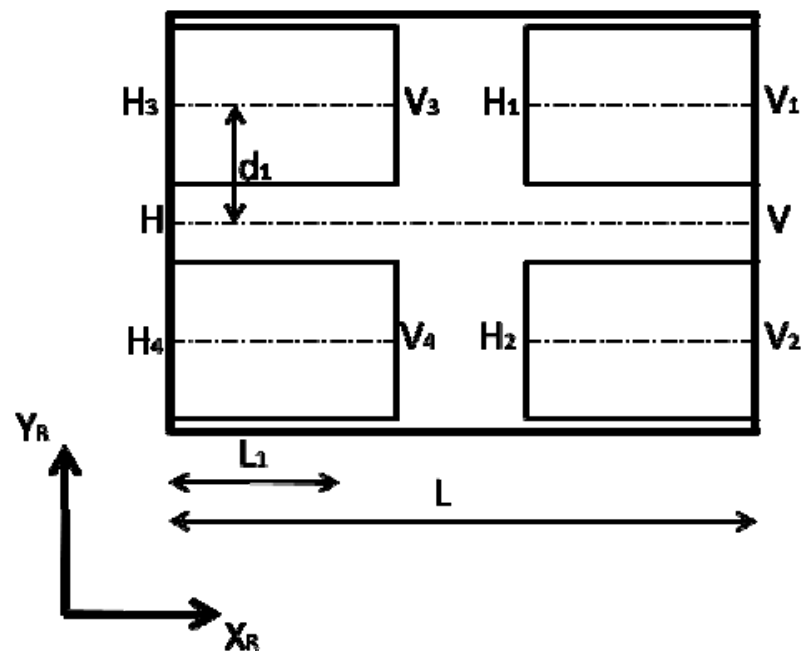

Abbildung 5: Anordnung der Element unter der Palette

Dieser Verbund soll einer einfachen Bahn folgen wobei die notwendigen Bewegungen der einzelnen Fahrzeuge unter der Palette beobachtet werden. Für eine einheitliche Darstellung wird ein globales, raumfestes Koordinatensystem $X_{R}, Y_{R}$ mit dem Ursprung in $\mathrm{O}(0,0)$ definiert. Des Weiteren werden die Punkte $\mathrm{V}$ und $\mathrm{H}$ auf der Palette definiert. Durch die Lage von $V$ und $H$ in der Ebene sind die exakte Position und Ausrichtung der Palette bekannt. Die Bahn die betrachtet wird setzt sich aus zwei Segmenten zusammen. Das erste Teilstück ist eine Gerade die auf der negativen $X$-Achse vom Koordinatensystem liegt, das zweite ist der positive Teil einer Parabel: 


$$
y=f(x)=\left\{\begin{array}{cc}
0 & x<0 \\
a x^{2} & x \geq 0
\end{array}\right\}
$$

In einer ersten Betrachtung wird der Punkt $\mathrm{V}$ mit der Bedingung entlang der Bahnkurve geführt, dass die Mittellinie immer Parallel zur Tangente der Bahn verläuft. Dies ist vergleichbar mit dem Fahren auf einem Linienleiter oder einer optischen Spurführung. Stellt man die Wegstrecke der einzelnen Punkte in einem Diagramm dar, wird deutlich dass nur für die Punkte auf der Vorderkante der Palette der Übergang von der Geraden- zur Kurvenfahrt glatt verläuft (Abb. 6). Die Punkte weiter hinten auf der Palette müssen abrupt ihre Richtung ändern. Die Rechtecke verbinden die Punkte zu diskreten Zeitpunkten und dienen lediglich der besseren Veranschaulichung.

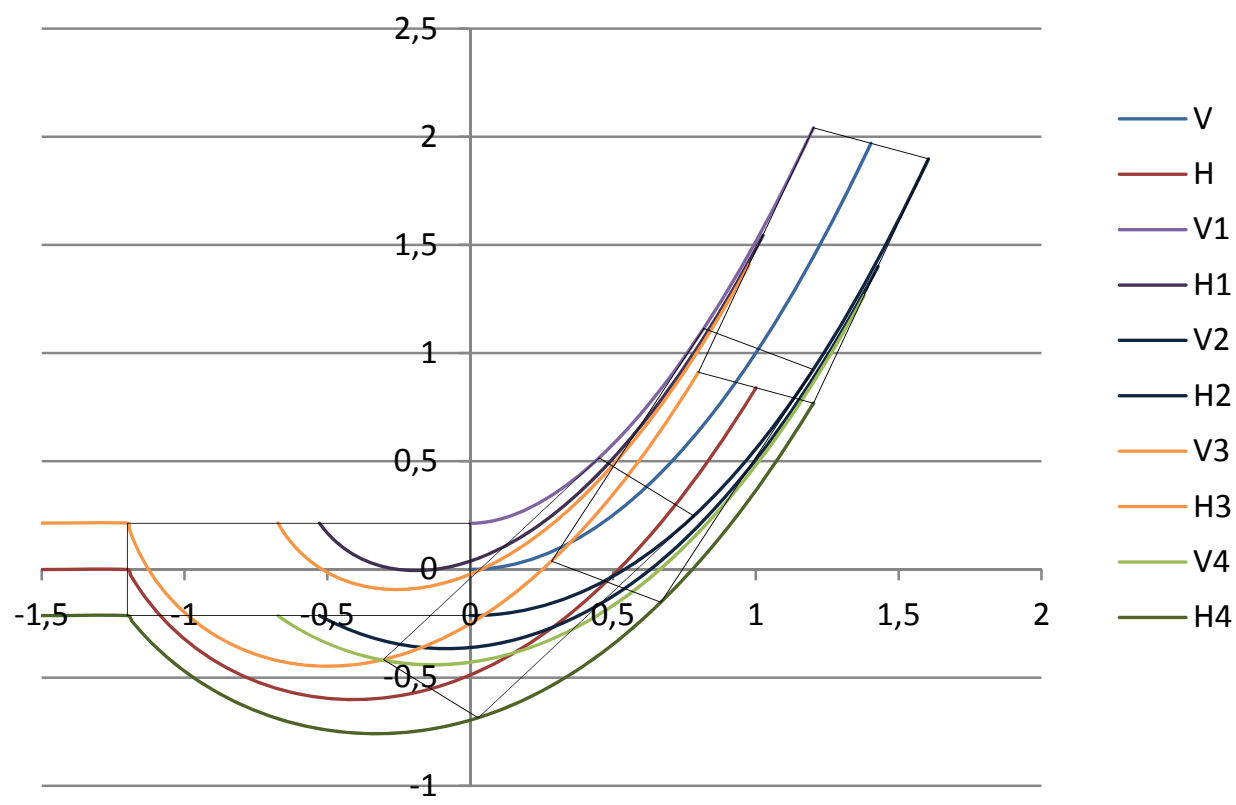

Abbildung 6: $V$ folgt der vorgegebenen Bahn

Unstete Fahrkurven einzelner Punkte treten immer dann auf, wenn ein Punkt der Fahrkurve folgt und dabei eine parallele Ausrichtung der Mittellinie zur Tangente eingehalten wird. Die Varianten, dass der Punkt $\mathrm{H}$ oder der Mittelpunkt des Verbundes der Fahrkurve folgt, verlagert das Problem lediglich auf andere Bereiche. In allen drei Fällen müssen gewisse Punkte auf der Palette ihre Richtung schlagartig ändern. Solche Fahrmanöver sind aufgrund der Dynamik auch mit holonomen Fahrwerken nicht umsetzbar. Bessere Ergebnisse lassen sich erzielen, wenn sowohl $\mathrm{V}$ als auch $\mathrm{H}$ der Bahnkurve folgen. In diesem Fall wird die Bedingung, dass die Mittellinie immer parallel zur Tangente verlaufen muss, aufgehoben. Mit dieser Veränderung können abrupte Richtungswechsel der Punkte vermieden und so eine praktische Umsetzung möglich gemacht werden (Abb. 7). Als nächstes werden die Geschwindigkeitsverläufe für eine solche Fahrt ohne abrupte Richtungswechsel betrachtet. 


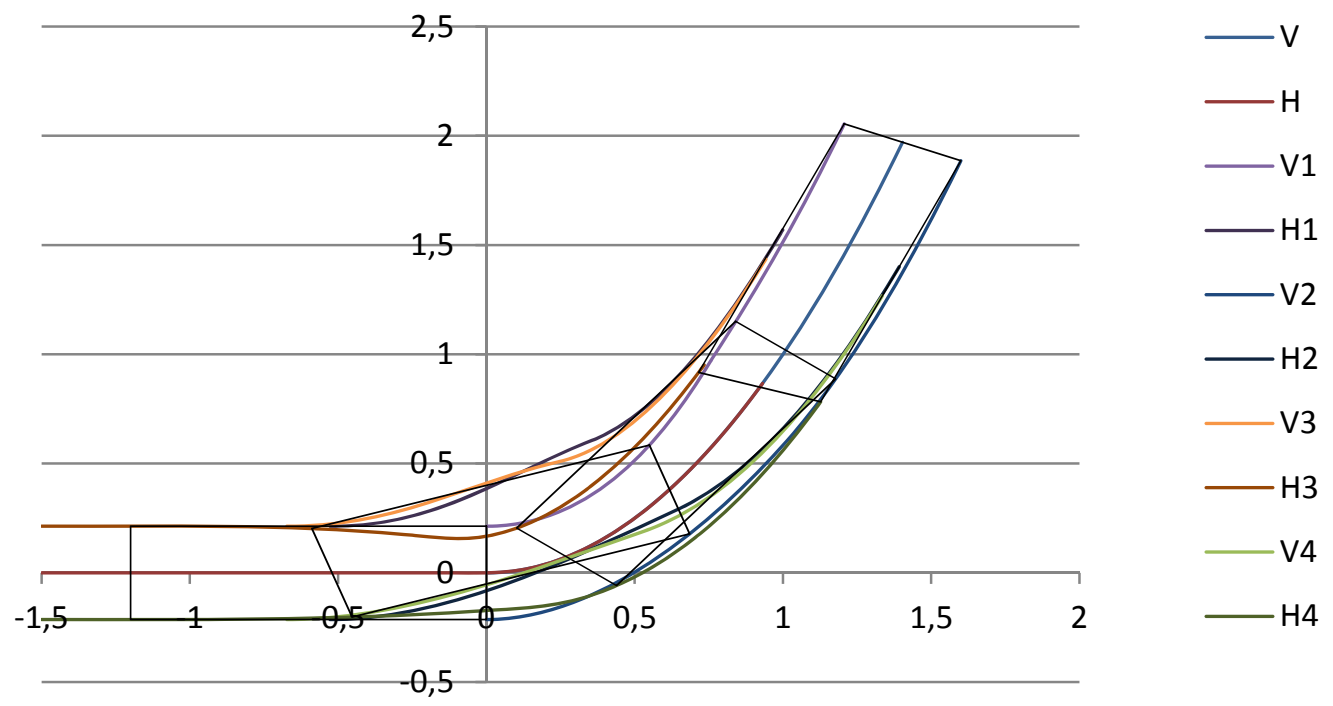

Abbildung 7: Bahnen der einzelnen Punkte auf den Fahrzeugen und der Palette

Es wird angenommen, dass sich $\mathrm{V}$ mit konstanter Geschwindigkeit entlang der Bahnkurve bewegt. Betrachtet man die Geschwindigkeitsverläufe der übrigen Punkte in Abhängigkeit dazu, so fallen abrupte Änderungen auf (Abb. 8).

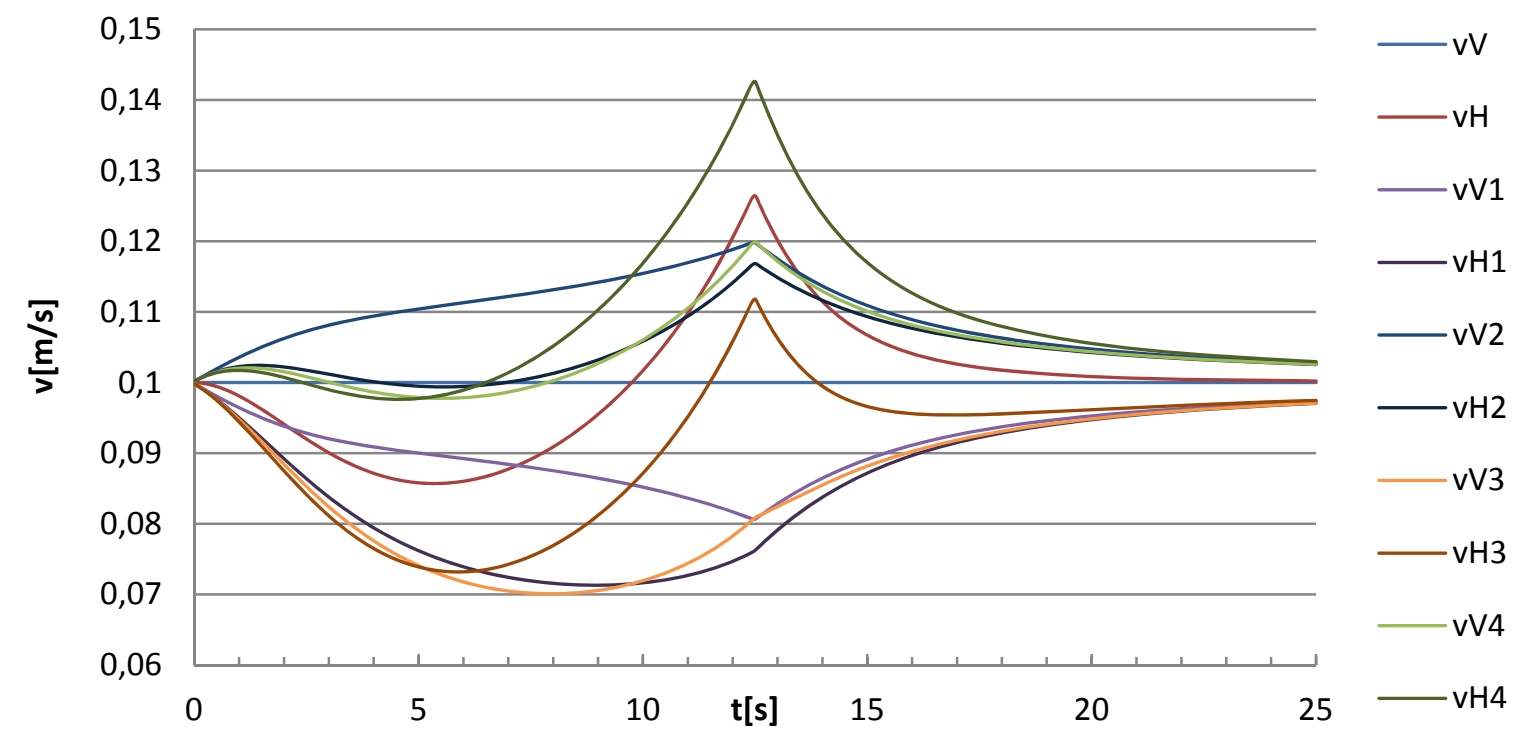

Abbildung 8: Geschwindigkeitsverlauf

Insbesondere im Zeitpunkt des Übergangs der Geradeausfahrt in die Kurvenfahrt treten bei $\mathrm{H}$ sehr hohe Beschleunigungen auf. Dies führt zu erhöhtem Schlupf und erhöhtem Verschleiß. Um bei Verbundfahrten diese Probleme zu vermeiden, bietet es sich an Fahrmuster aus Kurvenstücken und Geraden aufzubauen. Damit können Unstetigkeiten entlang der Strecke, sowohl bei den Fahrkurven als auch bei den Geschwindigkeitsverläufen, vermieden werden.

\section{$4 \quad$ Flächenbewegliche Fahrantriebe für moderne Materialflusssysteme}

In der VDI 2510 sind unter den flächenbeweglichen Antrieben drei Beispiele genannt, die in KARIS umgesetzt wurden.

(C) 2010 Logistics Journal : Proceedings - ISSN 2192-9084 
Zum einen der Topfantrieb, wie er auch schon in einem früheren Projekt (BiNE) zur Anwendung kam. Hierbei handelt es sich um mehrere unabhängige Fahr/Lenkeinheiten, womit Bewegungen in jeder Richtung möglich werden (Abb. 9).

Als nächstes die Radseitenlenkung mit einem zusätzlichen Freiheitsgrad um die Hochachse. Damit lässt sich der Aufbau beliebig über dem Fahrwerk ausrichten, um so ein Fahren in jede Richtung zu ermöglichen (Abb. 9).

Zuletzt der Mecanum-Antrieb, welcher als holonomer Antrieb (ähnlich einem OmniWheel) allein durch die Drehzahlunterschiede der Räder eine Flächenbeweglichkeit ohne Lenkbewegungen ermöglicht. Dieser Antrieb wurde am IFL als Kleinserie aufgelegt und so in 7 Testelementen zum Einsatz gebracht (Abb. 9).

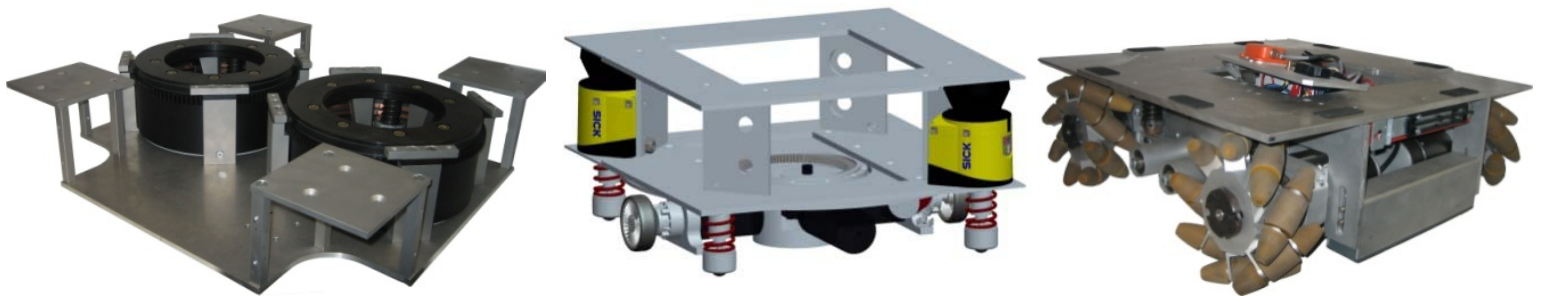

Abbildung 9: Topfantrieb, Radseitenlenkung, Mecanum-Antrieb

Darüber hinaus gibt es aus dem Bereich der Forschung noch einige andere Ansätze und Weiterentwicklungen für flächenbewegliche Antriebe. Einerseits eine Entwicklung aus Dortmund, welche auf dem aus dem Schiffsbau bekannten VoithSchneider-Antrieb beruht und andererseits diverse Weiterentwicklungen, speziell aus Japan, auf dem Bereich der OmniWheels. So sind z.B. Omniketten oder die OmniDisk entstanden.

\section{$5 \quad$ Untersuchung eines holonomen Fahrantriebes für KARIS}

Bei KARIS wurde aufgrund der Anforderungen der Mecanum-Antrieb gewählt. Die Anforderungen an Steuerbarkeit der Einzelmodule bei der Verbundfahrt sind sehr hoch und nur mit einem holonomen Antrieb mit der notwendigen Dynamik umsetzbar.

Im Folgenden wurde untersucht wie sich der aktuelle Antrieb genau verhält in puncto einhalten der Sollvorgaben. Im Gegensatz zu herkömmlichen Herangehensweisen aus der Informatik, die sich mit Formationsfindung und Formationsfahrt beschäftigen, kommen bei einem gemeinschaftlichen Transport weitere Randbedingungen dazu. So muss berücksichtigt werden, dass die Fahrzeuge ihre Position zueinander nicht verändern können und Abweichungen einzelner sich direkt auf den Verbund auswirken. 


\subsection{Versuchsaufbau}

KARIS verfügt für die Navigation und Sicherheit standardmäßig über zwei Sicherheitslaserscanner deren Reichweite $30 \mathrm{~m}$ beträgt. Die verwendeten Motoren sind mit Hall-Sensoren ausgestattet und geben die Drehzahl über eine CANSchnittstelle an die Fahrzeugsteuerung zurück. Um eine Referenzmessung durchführen zu können wurde ein weiteres Lasermesssystem (NAV 200, Fa. SICK) installiert, welches im Gegensatz zu den bordeigenen Scannern nicht mit einem Konturenmapping, sondern mit Reflektormarken arbeitet. Die Positioniergenauigkeit dieses Systems ist laut Hersteller mit $4 \mathrm{~mm}$ spezifiziert. Die Winkelauflösung beträgt $0,1^{\circ}$ bei einem Winkelfehler von $0,1^{\circ}$. Der Arbeitsbereich beträgt zwischen $1,2 \mathrm{~m}$ bis 28,5m. Um möglichst gleichmäßige Ergebnisse auf der gesamten Fläche zu erzielen wurden nach Absprache mit dem Hersteller zylinderförmige Reflektoren mit einem Durchmesser von $80 \mathrm{~mm}$ verwendet. Diese wurden unregelmäßig und an allen Seiten der Versuchsfläche verteilt. Auf dem KARIS-Element wurde der NAV 200 installiert. Zu diesem Zweck wurde einem der Elemente der Förderantrieb entfernt und der Scanner mit Hilfe einer Adapterplatte mittig auf inm platziert. Die Energieversorgung erfolgt über die integrierte Stromversorgung.

\subsection{Versuchsdurchführung}

Um Informationen über die Genauigkeit der Fahrwerke zu erhalten wurden einfache Geradeausfahrten unterschiedlicher Länge gemacht. Die veränderlichen Parameter sind Geschwindigkeit, Beschleunigung, Fahrstrecke und Fahrrichtung. Um deren Einflüsse besser beurteilen zu können, wurden drei der Werte konstant gehalten, während der andere systematisch verändert wurde. Dabei entspricht die Fahrtrichtung $0^{\circ}$ einer Fahrt entlang der Y-Achse in unserem ortsfesten Koordinatensystem und die Fahrtrichtung $90^{\circ}$ einer Fahrt entlang der X-Achse.

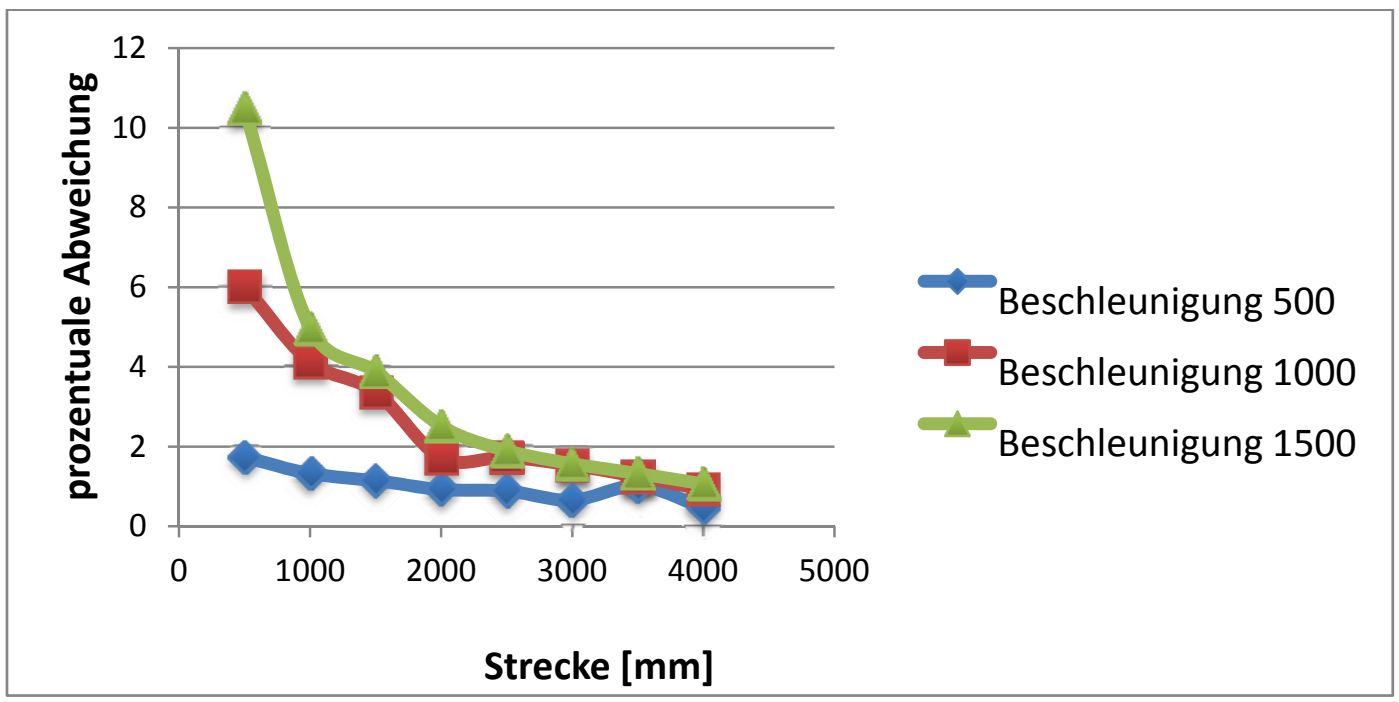

Abbildung 10: Prozentuale Abweichung beschleunigungsabhängig

Untersucht wurde zum einen welchen Einfluss die Beschleunigung in Abhängigkeit zur Fahrstrecke auf die Genauigkeit hat. Die prozentualen Abweichungen der Ist- zu 
den Sollwerten bei Fahrrichtung $0^{\circ}$ und Geschwindigkeit $1 \mathrm{~m} / \mathrm{s}$ sind in Abbildung 10 dargestellt. $\mathrm{Zu}$ erkennen ist, dass bei längeren Fahrten der Einfluss der Beschleunigung zurück geht und sich die Abweichungen einem Wert nähern. Um Einzelfehler auszuschließen wurde pro Punkt aus 30 Messfahrten ein Mittelwert gebildet.

Zum anderen wurde untersucht, wie sich die Fahrtrichtung des Antriebs auf die Genauigkeit auswirkt. Zu diesem Zweck wurden Testreihen gemacht, in denen die Fahrtrichtung und Fahrtstrecke bei konstanter Beschleunigung und Geschwindigkeit variiert wurden. Diese Fahrten wurden dann mit der Referenzfahrt (Fahrtrichtung = $0^{\circ}$ ) verglichen. Abbildung 11 zeigt den Vergleich der Richtungen bei Beschleunigung $\left(1 \mathrm{~m} / \mathrm{s}^{2}\right)$ und Geschwindigkeit $(1 \mathrm{~m} / \mathrm{s})$. Deutlich wird dabei, dass die Fahrtrichtung bis $45^{\circ}$ sehr gute Werte liefert, danach allerdings ein sprunghafter Anstieg der Ungenauigkeit zu verzeichnen ist.

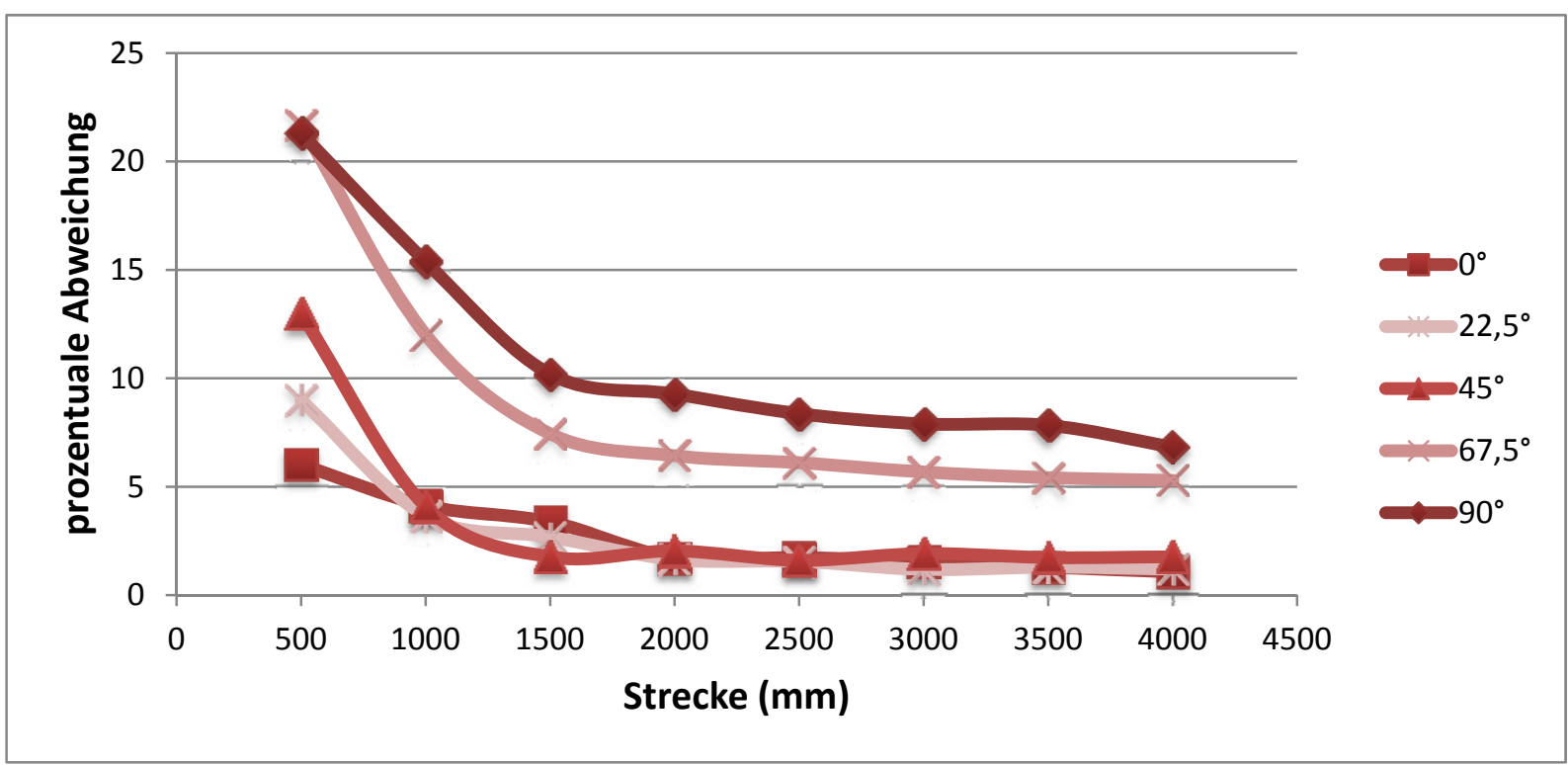

Abbildung 11: Prozentuale Abweichung richtungsabhängig

Betrachtet man die Fahrten über $3000 \mathrm{~mm}$ bei konstanter Geschwindigkeit und Beschleunigung, so fallen die unterschiedlichen Krümmungen der Fahrkurven hin zur Fahrt in $45^{\circ}$ Richtung auf. Dies ist in Abbildung 12 dargestellt. Interessant ist der Vergleich von Fahrten unterschiedlicher Länge bei ansonsten gleichen Parametern. Dabei wird der Einfluss von Beschleunigung und Verzögerung auf die Genauigkeit sichtbar, womit sich auch die prozentual größeren Abweichungen bei kurzen Fahrstrecken erklären. Die Vorgabe ist ein gerader Streckenverlauf, der sich im Diagramm nicht wiederfindet. Auffällig ist das konstante Verhalten, sobald sich das Element in gleichmäßiger Fahrt befindet (Abb. 13). Sehr gut zu erkennen ist das einheitliche Verhalten beim Beschleunigen und Verzögern. Der Bereich mit konstanter Fahrt läuft relativ parallel zur Sollrichtung und in jedem Fall gerade. Kritisch ist die seitliche Abweichung von der Sollstrecke. 


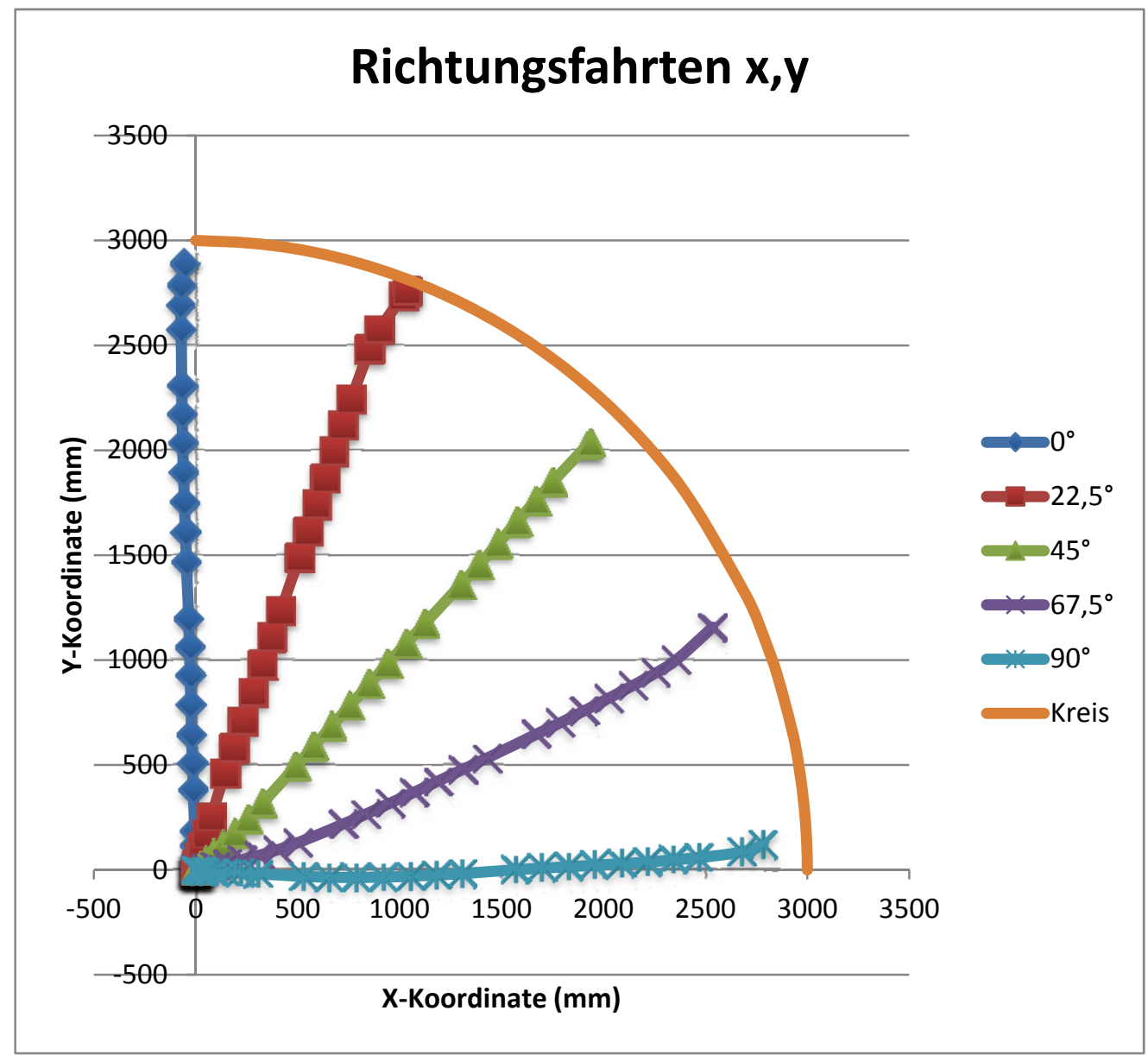

Abbildung 12: Vergleich Richtungsfahrten gleicher Länge

Die prozentualen Abweichungen (Abbildung 10 und 12) liegen zwar im niedrigen Prozentbereich, was allerdings keine Aussage Rückschlüsse darüber erlaubt, wie genau die Sollkurven eingehalten werden. Dies kann in der Praxis zu weiteren Problemen führen, da beim qualitativen Vergleich unterschiedlicher Fahrantriebe bei gleichen Sollvorgaben, für jeden Antrieb ein eigenständiges Verhalten während des Beschleunigens und Abbremsens beobachtet werden konnte.

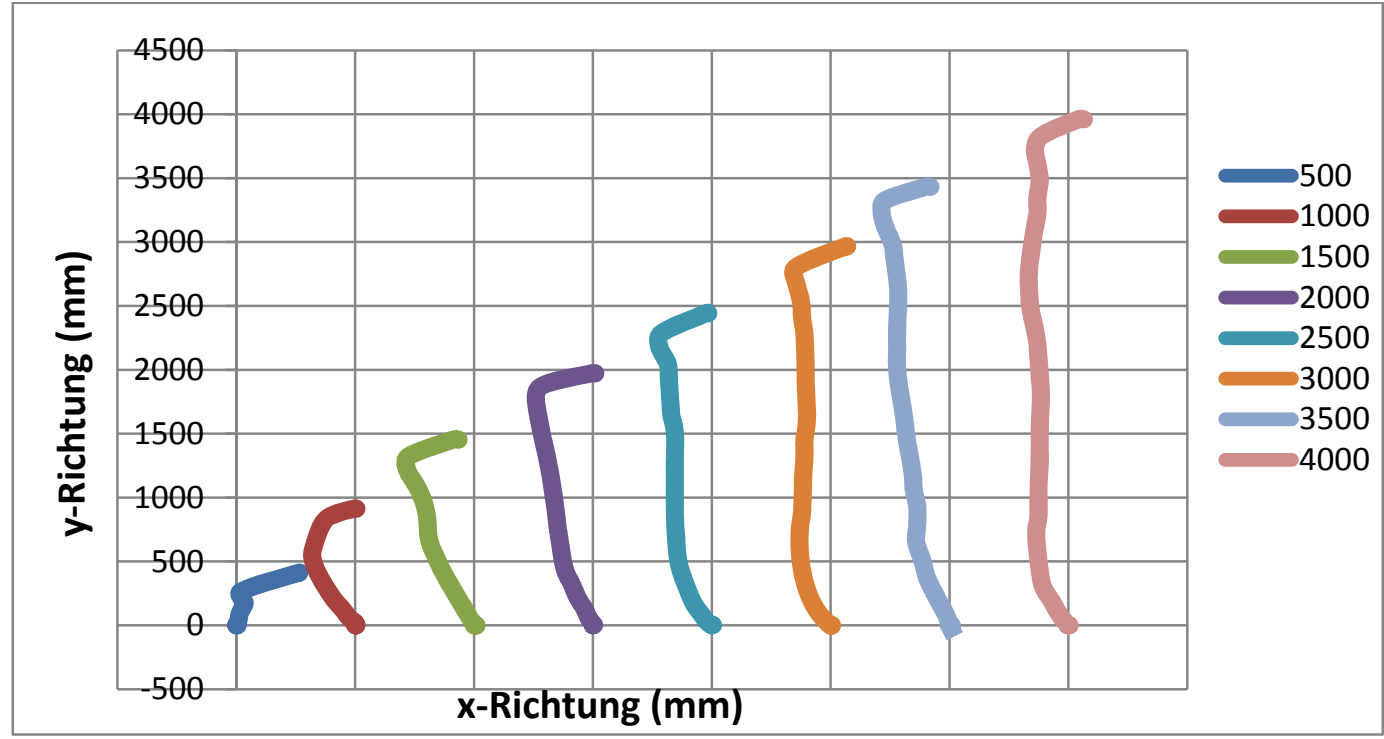

Abbildung 13: Vergleich Fahrten gleicher Richtung unterschiedlicher Länge 
Bei anderen Versuchen hat sich herausgestellt, dass die Fahrwerke unterschiedlich starke Haltekräfte in den jeweiligen Richtungen aufweisen. So war bei gespiegelter Zugrichtung $\left(-45^{\circ}\right.$ und $\left.+45^{\circ}\right)$ ein Unterschied von bis zu $100 \mathrm{~N}$ messbar. Diese Unterschiede und das damit verbundene Auswandern nach immer derselben Seite lässt sich auf Fertigungsungenauigkeiten zurückführen.

Vergleicht man den Mecanum-Antrieb der ersten Fahrzeuggeneration mit der jetzigen Entwicklungsstufe, so wird eine Verbesserung sichtbar. Betrug die prozentuale Abweichung beim ersten Fahrwerk noch deutlich über sieben Prozent (bezogen auf eine Fahrstrecke von 3000m hin und zurück), so waren es bei Fahrwerk Nummer drei nur noch knapp vier. Dies ist vor allem auf das reduzierte Spiel in der Radlagerung, den kugelgelagerten Rollen und der verbesserten WelleNabe-Verbindung zum Getriebe zurückzuführen. Die Versuche haben gezeigt, dass für eine Verbundfahrt eine Regelung notwendig sein wird. Die Abweichungen der Istzu den Sollwerten sind zwar gering, reichen jedoch aus um bei einer Verbundfahrt für erhöhten Verschleiß zu sorgen. Auch konnten in den Versuchen der Einfluss von Bodenunebenheiten, Steigungen und Verschmutzung nicht berücksichtigt werden, was zu einer weiteren Verschlechterung führen wird.

\section{Literatur}

[FWS10] Furmans, K., Wisser, J. und Schwab, M.: Kosten- und Leistungsstrukturen in Distributionszentren. In: F+H, H. 7-8, S. 257259. Mainz: Vereinigte Fachverlage, 2000.

[B03] Barthelemy, J.: The seven deadly sins of outsourcing. In: Academy of Management Executive, Vol. 17, No. 2, pp. 87 - 98, 2003.

[FSS09] Furmans, K., Schleyer, M. Schönung, F.: A Case for Material Handling Systems Specialized on Handling Small Quantities. IMHRC, 2009.

[NB10] Nobbe, C., Berbig, D.: VDI-IFL Sommerseminar 2010, Wege zu einer verantwortlichen Ressourcenverwendung in der Logistik, Ressourcen länger und flexibel nutzen. ISSN Nr. 2190-6599, 2010.

[FSG10] Furmans, K., Schönung, F., Gue, K.: Plug-and-Work Material Handling Systems, 2010.

[F01] Studie von Fraunhofer ISI, VDI-Nachrichten Nr. 035 vom 31.08.2001, S.03, Düsseldorf: VDI-Verlag, 2001.

[VDI05] VDI 2510, Fahrerlose Transportsysteme (FTS), Verein Deutscher Ingenieure e.V., Düsseldorf. Berlin: Beuth Verlag GmbH, 2005. 